\title{
Practical approaches to assessment of harmonics along radial distribution feeders
}

DOI:

10.1109/TPWRD.2019.2901245

\section{Document Version}

Accepted author manuscript

Link to publication record in Manchester Research Explorer

\section{Citation for published version (APA):}

Abdelrahman, S., \& Milanovic, J. V. (2019). Practical approaches to assessment of harmonics along radial distribution feeders. IEEE Transactions on Power Delivery. https://doi.org/10.1109/TPWRD.2019.2901245

\section{Published in:}

IEEE Transactions on Power Delivery

\section{Citing this paper}

Please note that where the full-text provided on Manchester Research Explorer is the Author Accepted Manuscript or Proof version this may differ from the final Published version. If citing, it is advised that you check and use the publisher's definitive version.

\section{General rights}

Copyright and moral rights for the publications made accessible in the Research Explorer are retained by the authors and/or other copyright owners and it is a condition of accessing publications that users recognise and abide by the legal requirements associated with these rights.

\section{Takedown policy}

If you believe that this document breaches copyright please refer to the University of Manchester's Takedown Procedures [http://man.ac.uk/04Y6Bo] or contact uml.scholarlycommunications@manchester.ac.uk providing relevant details, so we can investigate your claim.

\section{OPEN ACCESS}




\title{
Practical Approaches to Assessment of Harmonics Along Radial Distribution Feeders
}

\author{
Sami Abdelrahman and Jovica V. Milanović, Fellow, IEEE
}

\begin{abstract}
A high level of delivered power quality is becoming one of the key performance indicators for both contemporary and future power networks. The increased proliferation of converter connected generation and load in power networks will lead to increased levels of harmonics in distribution networks. A general knowledge of the distribution network performance in terms of harmonics is essential for distribution network operators for both operating and planning networks while considering power quality performance. The main limitation is the reduced monitoring infrastructure compared to transmission networks. This paper presents an efficient method for assessment of harmonic levels along medium voltage radial distribution feeders based on a single measurement at a substation. The methodology is illustrated in a case study involving a real life medium voltage 35 bus radial distribution feeder.
\end{abstract}

Index Terms - Coarse analysis, estimation, harmonics propagation, probabilistic analysis.

\section{INTRODUCTION}

$\mathrm{H}$ armonic sources are increasing rapidly in today's distribution networks. Under the current paradigm shift towards greener more efficient networks, power electronic interfaced loads and different generation and storage technologies are widely utilised. Convertor interfaced generators, storage and variable-speed drive (VSD) interfaced motors, for example, are sources of nonlinearity and distortion at the connection buses. Such types of equipment are usually manufactured and tested considering the standard limits of harmonic injections. Nevertheless, the aggregated effects of a high penetration of such loads and generators might lead to permanent or temporary harmonic standard limit violations at the connection points at the low voltage (LV) or even the medium voltage (MV) point of common coupling (PCC).

Standard limit levels regarding harmonic voltages and total harmonic distortion (THD) are defined in international standards [1, 2], however exact 'pain'/sensitivity levels vary between different types of equipment. Increased losses, premature aging, communication interference and equipment damage under resonance conditions are some of the wellknown impacts of excess harmonics. Most of these effects, however, are difficult to model and many uncertainties are

This work was supported by SuSTAINABLE Project under Grant 308755 . Sami Abdelrahman is with Nationalgrid plc., Warwick Technology Park Gallows Hill, Warwick, CV34 6DA UK and Jovica V Milanovic is with the School of Electrical and Electronic Engineering, The University of Manchester, Manchester, M13 9PL, UK. (email: milanovic@ manchester.ac.uk). involved in the estimation of customer and utility losses due to harmonics. Moreover, only a few regulators worldwide force harmonic limits or penalize Distribution System Operators (DSO) based on harmonic performance, and the limits are enforced based on a connection agreement between the customer/DG owner and the utility [3]. Thus, the network wide monitoring and the observability of harmonics levels throughout LV and MV distribution feeders are still very limited in most of today's distribution networks.

Harmonic State Estimation (HSE) is the process of establishing the levels of the harmonic voltages at all network buses based on a limited number of harmonic readings. For transmission systems it is a well-developed process [4-7]. Taking advantage of the high number of monitored buses, the symmetry of the network, the meshed topology and a prior knowledge of most probable location of harmonic sources, the HSE for transmission systems can give accurate estimates of harmonic levels and can pinpoint the harmonic sources by applying proven estimation mathematical models like the Weighted Least Squares (WLS) technique. More recent publication considered time-varying harmonics in the estimation [8]. It was also shown in $[9,10]$ that the time domain harmonic state estimation can be performed by applying new techniques and by utilising the advancement in computing hardware to reduce computational time.

On the other hand the HSE for distribution networks is still developing. The limited monitoring infra-structure, the limited knowledge of the status of some components (especially capacitors), the high variance and uncertainty in harmonics injections and locations, the high $\mathrm{R} / \mathrm{X}$ ratio of branches and the radial or weakly meshed topology complicate the problem of harmonic estimation. The radial topology and limited number of monitors in addition to a high variance of harmonics through long periods of study complicate the problem of the optimum positioning of monitors to obtain full observability. These aspects of distribution feeders usually lead to difficulties in getting independent readings, which in turn leads to ill-conditioned matrices for both estimation and monitors locating problems and causing, in general, solutions convergence issues [4]. However, on the positive side, the simple topology of distribution feeders lends itself to simplified calculations and the possibility of the direct application of Kirchoff's Laws to gain useful information. Recent references discussed the HSE problem for distribution networks and suggested solutions like monitoring certain harmonic frequency or utilising synchronised measurements from Phasor Measurement Units (PMUs) [11-13]. 
References $[14,15]$ present a technique for the estimation of harmonic levels and provide an educated guess for the most probable location of harmonic sources. This algorithm requires two or three monitors located at nodes that divide the feeder into sections. Based on the fact that harmonic currents flow towards the sub-station [14], the most probable location of non-linear sources can be associated with certain sections of the feeder. A good estimate of overall harmonic levels can be provided. Also, it was shown in [16] that a high level of knowledge about radial distribution feeders harmonic performance can be achieved based on substation readings. While extra monitors and field measurements can help to pinpoint sources and increase accuracy, they are expensive and not always permanently located at distribution feeders [16].

This paper presents two efficient methodologies for harmonic analysis and assessment in distribution feeders. The proposed methodologies are not based on the state-estimation concept but aim to provide approximate evaluation of the harmonic performance in radial distribution feeders with limited monitoring. Based only on the general knowledge of load types, network parameters and single monitor at the substation; approximation of the available harmonic distortion capacity along the feeder can be estimated using developed look-up curves. Furthermore, the proposed probabilistic methodology can provide a more accurate assessment of the THD and individual harmonic voltage levels over a given time period at all buses along the feeder. A case study based on a 35-bus real distribution feeder is used to illustrate developed approaches. All simulations are performed in OpenDSS and MATLAB environments.

\section{HARMONICS IN RADIAL FEEDERS}

There are well developed techniques for the harmonic analysis of radial distribution feeders. Usually an analysis of expected low harmonic frequencies and potential resonance at the feeders is adequate in order to identify any unacceptable performance, and where further analysis with more accurate modelling will be required [17].

Based on the fact that the harmonic currents flow towards the substation; a rough estimate of THD levels can be produced based on the knowledge of the feeder parameters and the harmonic readings at the substation. This assessment of feeder harmonic performance can provide a look-up figure indicating the remaining THD capacity of the feeder based on the THD recorded at the sub-station. The THD capacity can be used in distribution planning considering PQ performance. As discussed in [18] for calculating the remaining load growth permissible before reaching harmonic limits, or in more recent studies as in $[19,20]$ to determine the level of permissible penetration of distributed generation.

For capacitor free feeders with the assumption of no harmonic current cancellation, the minimum THD of the feeder is at the substation bus [21]. Therefore, the THD along the feeder usually increases with the electrical distance from the substation, i.e., with higher system impedance or lower shortcircuit capacity, see Figure 1. The current practice of evaluating new connections under stages 2 and 3 of [3] is to calculate the THD headroom at the connecting point and neighbouring buses. However, for radial distribution feeders with comparable load characteristics, the shape of the THD vs. feeder length is exponential with the growth constant directly related to the electrical distance. Therefore, the increase of THD at a certain point will shift the whole curve up and most probably bringing the nodes further from the sub-station closer to the planning level. The harmonic analysis presented in this paper is applicable to radial feeders with comparable load characteristics.

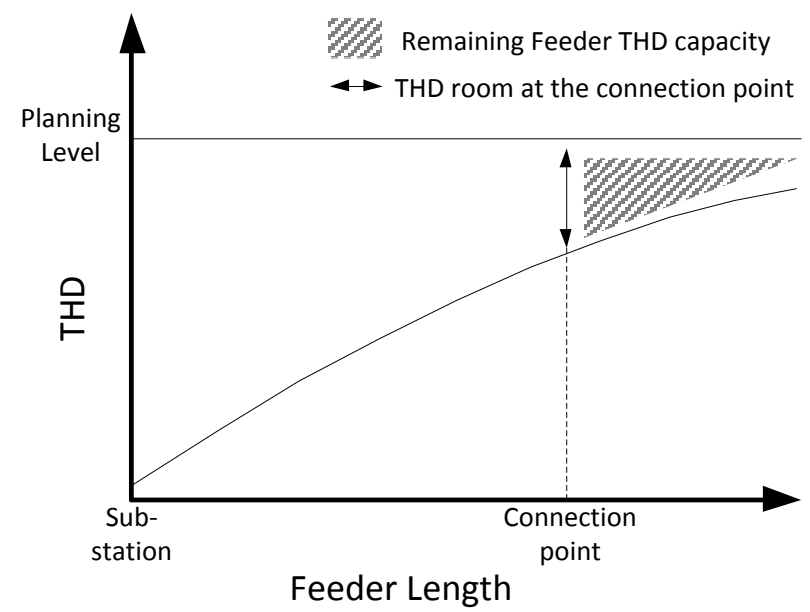

Fig. 1. THD increases with the feeder length

\section{TeSt NeTworK AND CASE STUdIES}

\section{A. Test Network}

The test network adopted for the harmonic simulation over one day is a real 35 -bus medium voltage $(15 \mathrm{kV})$ distribution feeder connected to the external grid through $60 / 15 \mathrm{kV}$ Yy transformer. Thirty buses are load buses with both commercial and domestic types of load connected. The total peak load at the study day is $3.1 \mathrm{MW}$ and 1.1 MVAr. The displacement power factor was kept constant for all load buses at 0.94 .

Different types of cable are installed, with $\mathrm{R} / \mathrm{X}$ ratio ranging between 1.75 and 5 , the total length of feeder branches is around $8 \mathrm{~km}$. All load buses are considered to have harmonic sources with different levels and frequencies throughout the study period. Also, a zero sequence path was provided by connecting sub-station transformer windings and loads as grounded star, to analyse the flow of triplen harmonics. Further details about the test feeder can be found in $[22,23]$.

Six characteristic buses were adopted for analysis; Bus 01, the MV bus of the substation (monitored), Bus 06, Bus 15 and Bus 28 at different locations on the main path, Bus 19 at the end of a small branch and Bus 35 at the end of the main path, see Figure 2. Table I shows the fundamental frequency electrical distance of the selected buses from the monitored bus, Bus 01.

TABLE I. CHARACTERISTIC BUSES DiSTANCE

\begin{tabular}{|l|c|l|c|}
\hline Bus & Electrical distance $(\Omega)$ & Bus & Electrical distance $(\Omega)$ \\
\hline Bus 06 & $0.702+\mathrm{j} 0.302$ & Bus 28 & $2.442+\mathrm{j} 0.975$ \\
\hline Bus 15 & $1.400+\mathrm{j} 0.572$ & Bus 35 & $4.389+\mathrm{j} 1.732$ \\
\hline Bus 19 & $1.707+\mathrm{j} 0.692$ & \multicolumn{3}{|c}{} \\
\cline { 1 - 3 } & &
\end{tabular}




\section{B. Superposition case study}

The superposition case study was performed by equal harmonic injections at the characteristic buses (except Bus 01) one bus at a time, and the propagation of the harmonic distortion was studied. From Figure 3 it can be seen that the injected harmonics resulted in THDv at the injecting bus that propagates un-attenuated towards the end of the feeder. This is simply because at the injection point the path towards the substation provides the low impedance path while the opposite direction is effectively an open circuit for harmonic voltages. The harmonic voltages drop in the direction of the substation is almost equal for all five cases and directly related to the network harmonic impedance. Therefore, the THD at the substation is the same for all the studied injection buses. Note the special case of harmonic injection at Bus 19 (red line-dot markers) as the bus is at the end of a branch (Figure 2) and not in the main feeder. After subtracting the THD drop in the branch, however, the THD values at buses 15, 28 and 35 are equal.

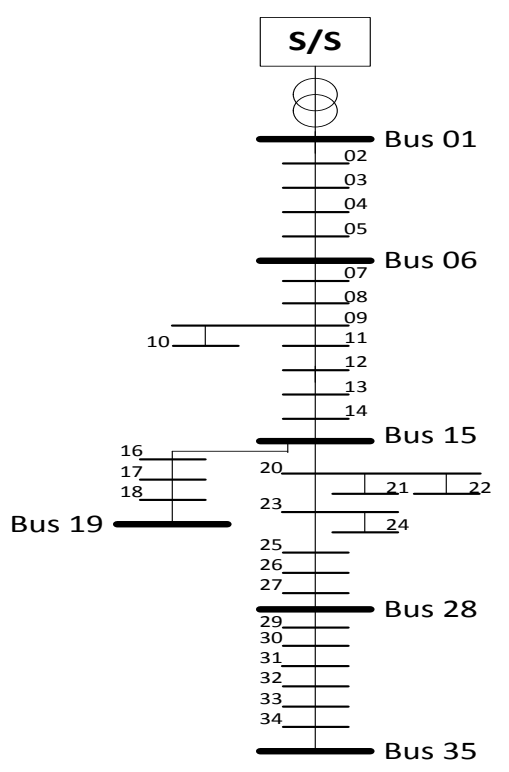

Fig. 2. Test feeder ( $\mathrm{S} / \mathrm{S}$ is $60 / 15 \mathrm{kV}$ substation)

To study the propagation of the THD rather than separate harmonic voltages, the electrical distance of characteristic buses to the substation bus at different frequencies were aggregated. The total harmonic electrical distance was produced by the weighted sum of electrical distances at $N$ considered harmonic frequencies and $i$ buses. The $h^{\text {th }}$ harmonic impedance is weighted by the ratio $1 / \mathrm{h}$, based on the expected harmonic currents flowing at that harmonic frequency, equation (1). The $\mathrm{Z}_{\mathrm{THD}}$ is normalized based on the maximum harmonic electrical distance calculated for a bus, which is normally indicating the physically furthest connected bus from the substation for radial feeders.

$$
Z_{T H D, i}=\sum_{h=2}^{N} \frac{Z_{h, i}}{h}
$$

By applying the concept of the total harmonic electrical distance for the combined results (average THD at the bus when injection is at the same bus) the linear relationship between $Z_{T H D}$ and the THD propagation can be noticed in Figure 4 . The feeder electrical length is normalized based on the furthest bus, Bus 35, i.e., $\left|Z_{T H D, 35}\right|=11.13 \Omega$, as shown in Table II.

TABLE II. TOTAL AND INDIVIDUAL HARMONIC ELECTRIC DISTANCES FROM SUBSTATION (BUS 01) IN OHM

\begin{tabular}{|c|c|c|c|c|c|c|c|c|}
\cline { 2 - 9 } \multicolumn{1}{c|}{} & $\left|\mathrm{Z}_{1}\right|$ & $\left|\mathrm{Z}_{3}\right|$ & $\left|\mathrm{Z}_{5}\right|$ & $\left|\mathrm{Z}_{7}\right|$ & $\left|\mathrm{Z}_{9}\right|$ & $\left|\mathrm{Z}_{11}\right|$ & $\left|\mathrm{Z}_{13}\right|$ & $\left|\mathrm{Z}_{\mathrm{THD}}\right|$ \\
\hline Bus 06 & 0.76 & 1.14 & 1.61 & 2.12 & 2.64 & 3.17 & 3.71 & 1.87 \\
\hline Bus 15 & 1.51 & 2.21 & 3.11 & 4.07 & 5.09 & 6.14 & 7.25 & 3.62 \\
\hline Bus 19 & 1.84 & 2.69 & 3.76 & 4.92 & 6.13 & 7.38 & 8.69 & 4.37 \\
\hline Bus 28 & 2.63 & 3.82 & 5.35 & 7.02 & 8.79 & 10.66 & 12.67 & 6.26 \\
\hline Bus 35 & 4.72 & 6.81 & 9.52 & 12.47 & 15.60 & 18.90 & 22.42 & 11.13 \\
\hline
\end{tabular}

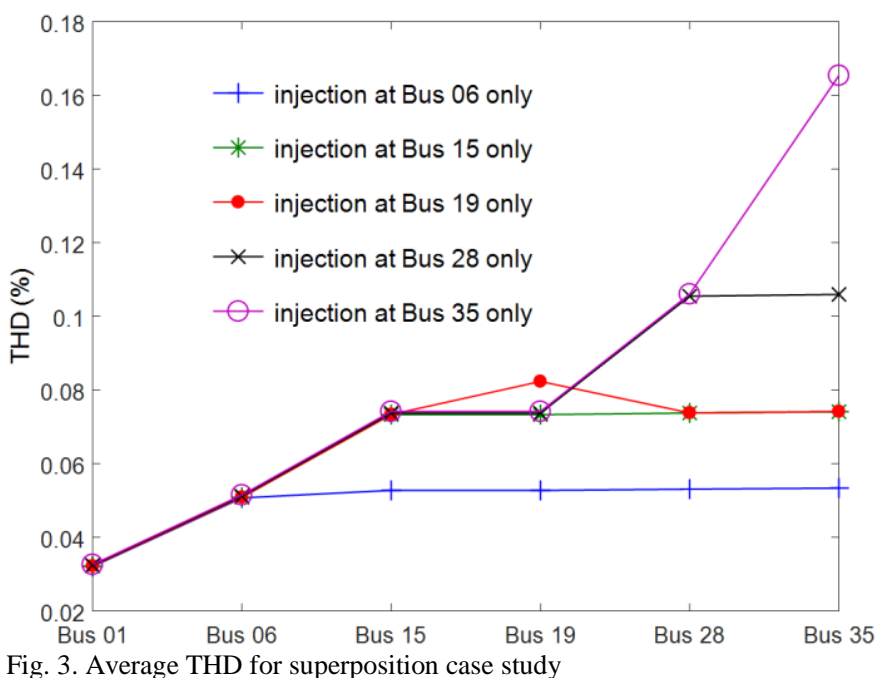

Fig. 3. Average THD for superposition case study

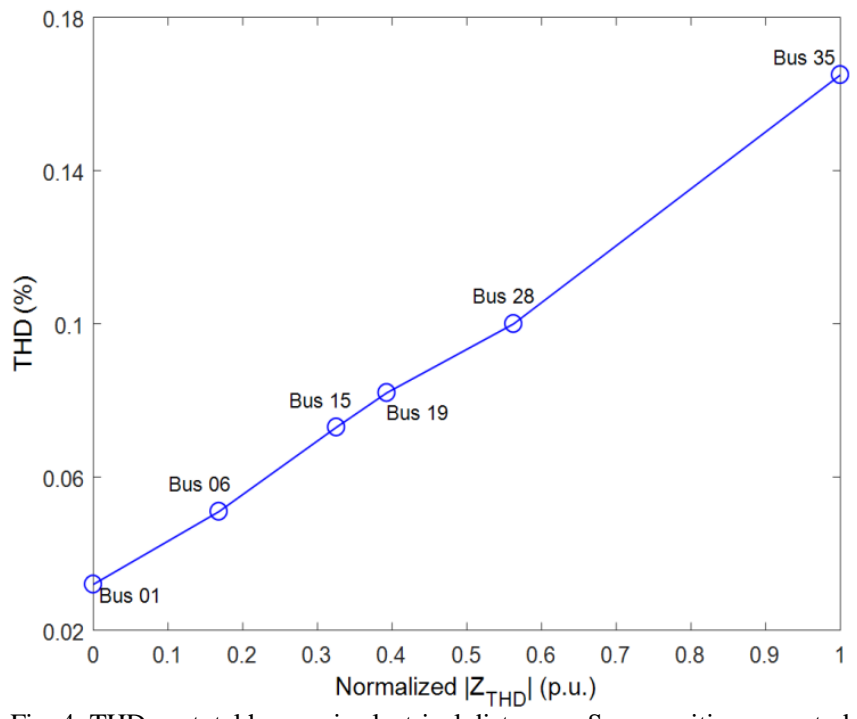

Fig. 4. THD vs. total harmonic electrical distance - Superposition case study

\section{Generic case study}

The generic case study is simulated to analyse the propagation of harmonics for known distorting loads. This was performed by adopting equal in-phase harmonic spectra for all loads using the theoretical values of convertors' harmonic currents [24]

$$
I_{h}=\frac{I_{1}}{h}
$$

Then by calculating the harmonic distortion for the 
characteristic buses for one day, considering the loading levels as the only varying parameter, the THD vs. total harmonic electrical distance curve was produced. Figure 5 shows the minimum, average and maximum THD value recorded during the day plotted against harmonic electrical distance $\mathrm{Z}_{\mathrm{THD}}$. The relationship between THD and $\mathrm{Z}_{\mathrm{THD}}$ is not linear in this case due to interaction between different sources of harmonic currents, i.e., a certain level of harmonic cancellation. In spite of injection at a $0^{\circ}$ phase shift from fundamental currents at each node, the harmonic phase angles at different summation points differ due to different harmonic impedances between the nodes. As shown in the figure, the THD tends to increase with the distance from the substation, also the variation range at the further buses is higher. This can be explained by the faster increase of impedance at the further buses with frequency as shown in Figure 6.

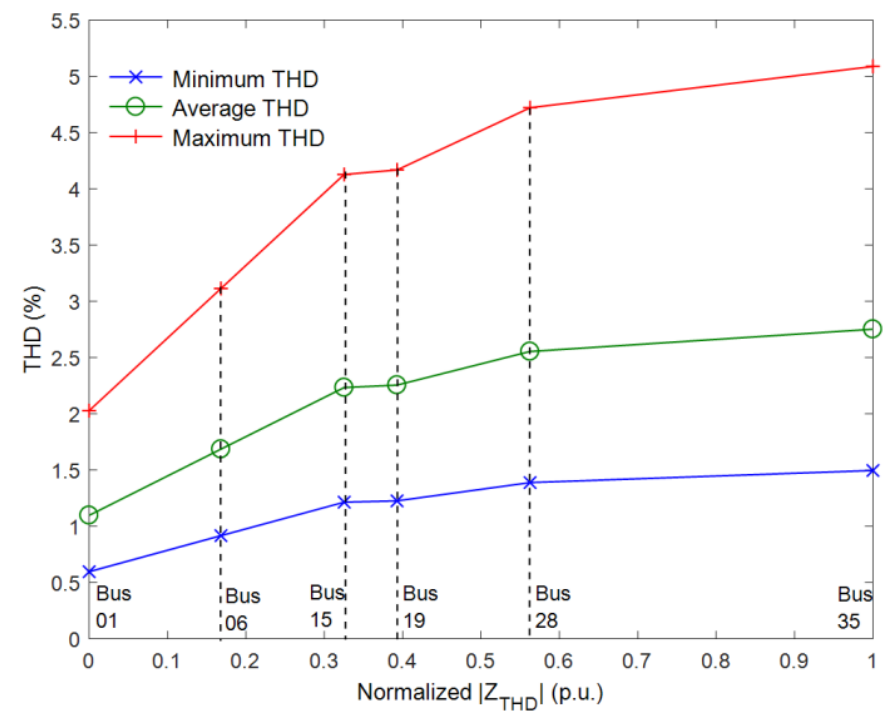

Fig. 5 THD vs. total harmonic electrical distance - Generic case study

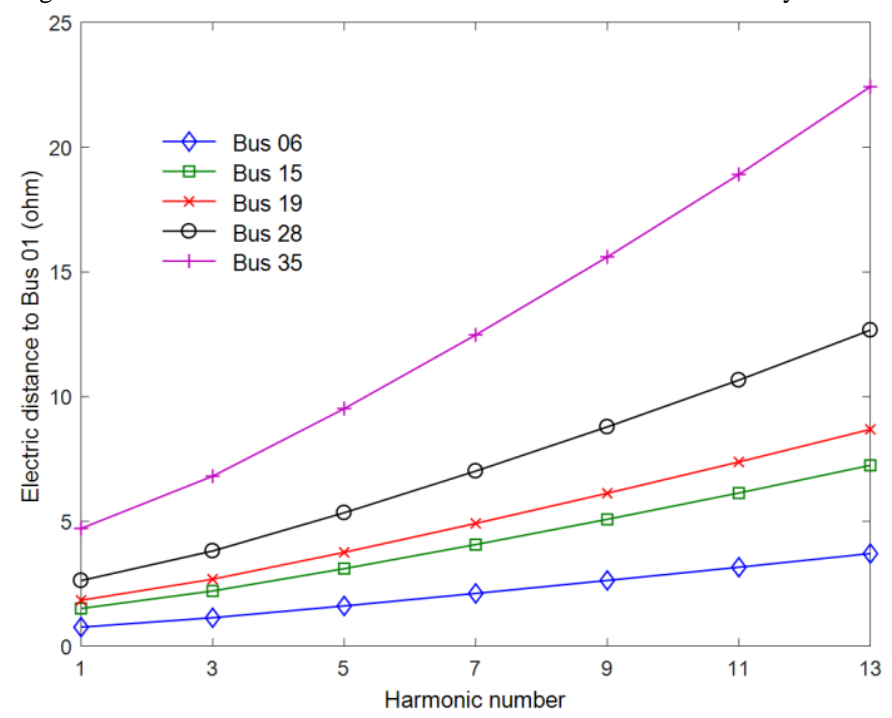

Fig. 6. Buses electrical distance per harmonic frequencies

\section{General case study}

A general case study was produced for a one day harmonic performance of the network, and used as a benchmark for the assessment. Two loading curves, for commercial and domestic loads, were adopted with a resolution of half an hour. Every half hour three harmonic voltages and current readings (representing a reading every ten minutes) were recorded, by sampling injections at all loading nodes from predefined average values of harmonic currents at each given node, and harmonic load flow was run. Every six hours the average values of harmonic injections and harmonic frequencies were varied representing the new composition of non-linear loads connected at the bus. The first six odd harmonic frequencies were considered, i.e. 3rd, 5th, 7th, 9th, 11th and 13th, with injection levels varying between $0 \%$ and $90 \%$ of fundamentals at different times and at different nodes. The THD ranged (for all buses) between $0.14 \%$ and $6.34 \%$ throughout the study day. Figure 7 shows the minimum, average, $95^{\text {th }}$ percentile and the maximum values of THD of the characteristic buses. Just as in the generic case study, the variation in the recorded THD increases with the distance from the substation.

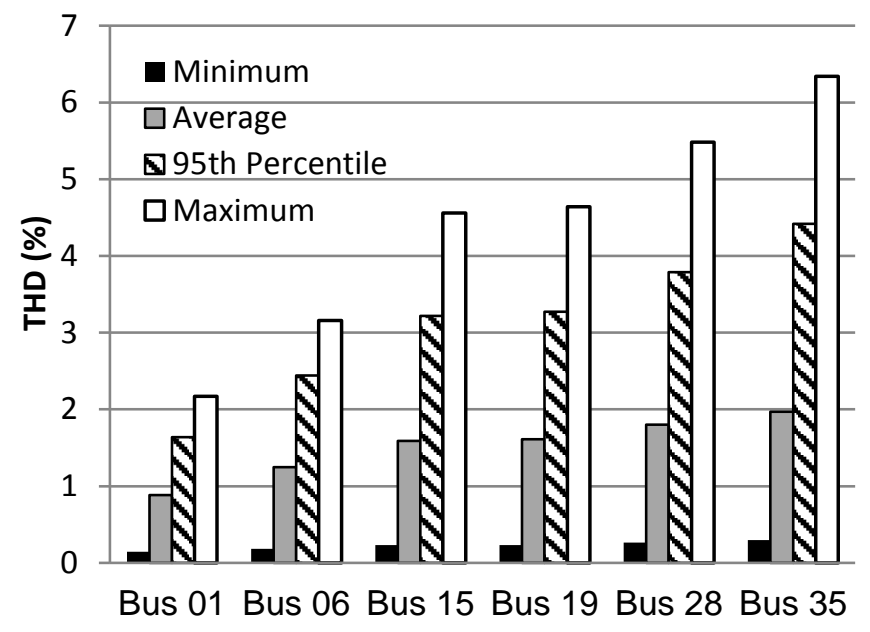

Fig. 7. General Case study results

\section{METHODOLOGY}

\section{A. Coarse Analysis}

The analysis is performed based on the generic case study presented in III.C. The curves of THD vs. harmonic electrical distance from Figure 5 are smoothed and used to perform two types of studies:

- The impact of increased injection at one node on the rest of the feeder.

- A rough assessment of THD along the feeder based on a substation harmonic reading.

The impact of the increased injections was performed in the generic case study. At Bus 06 the injection was increased to 2, 3 and 4 times the original injections, while the rest of the injecting loads were kept at the original values used in the generic case study. A one day harmonic evaluation was performed and the minimum, average and maximum THD values were plotted for the characteristic buses, as shown in the next section.

The second study was to perform a rough estimate of the general case study performance based on the generic average curve of THD vs. electrical distance. This was done by rescaling the THD curves from the generic case by a 
correction factor based on the THD values recorded for the general case study. Also, the variation level (measured by the coefficient of variation $\sigma / \mu$ ) measured at the substation for the general case was used to estimate other indices, i.e. maximum and $95^{\text {th }}$ percentiles of THD.

\section{B. Probabilistic assessment}

The assessment of harmonic levels is performed based on the methodology described in $[14,15]$. However, the main aim here is to estimate the levels of harmonic distortion rather than pinpointing the harmonic sources themselves. Based on the fact that all harmonic currents flow towards the sub-station [14] in normal conditions, monitor readings at the sub-station should provide basic information about the frequencies and the levels of harmonic current flow throughout the feeder, assuming that there is no high level of harmonic cancellation or harmonics diverted to the ground through capacitors or filters, Figure 8 shows an example where a $5^{\text {th }}$ harmonic current is present at some of the feeder branches but might not be recorded by the substation monitor due to a capacitor installed along the feeder.

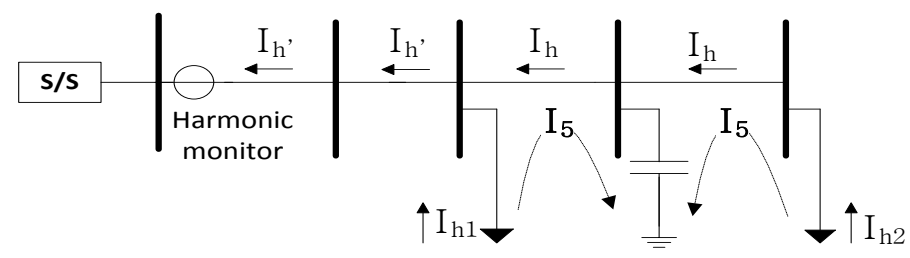

Fig. 8. A case of $5^{\text {th }}$ harmonic current not seen at the substation

The developed algorithm is based on Monte Carlo (MC) simulations of the injection levels at all nodes. For the MC simulation the sampling range and the number of simulations should be selected based on the expected variation of harmonic performance during the study period. A number of uncertainties are involved in the simulation of harmonic levels. Uncertainties like loading levels, injection levels and angles affect the shape and the range of the samples of results of harmonic voltage and THD. For example, considering the generic case study (changing only load levels with all remaining parameters fixed) harmonic daily performance, the THD variation during the day is approximately equal to the characteristic buses with a coefficient of variation $\sigma / \mu=0.35$. This indicates a daily variation range of more than $\pm 100 \%$ of the average value for $3 \sigma$ covering $99.7 \%$ of observations. To perform the MC based on such a high range, a high number of simulations is required. Therefore, to exclude the loading variation from the assessment, predefined loading curves based on the knowledge of the day type (weekend/weekday) and the load type (domestic/commercial) are applied at different nodes, and the estimation function is modified to include time-stamped readings. By estimating a reading at a time, a range of $\pm 30 \%$ of average with only 100 harmonic simulations yields an estimation of $95^{\text {th }}$ percentiles of THD values with an average error of $6 \%$ for the characteristic buses.

The recorded spectra at the substation are taken as the initial estimate of the injections at all nodes, i.e., the ratio of harmonic currents to fundamentals recorded at the substation is assumed to be relatively contributed to by all loads based on their size. These values are adopted as the mean values of the injection samples. Then, by randomly sampling normally distributed harmonic injections around these mean values with constant angles based on the monitored values, a number of harmonic load flows are run and the $\mathrm{V}_{\mathrm{h}}$ and THD values at all nodes are calculated. The final assessment/estiamte is produced by comparing the substation (monitor location) harmonic voltages $\mathrm{V}_{\mathrm{h}}$ obtained by every simulation with the recorded monitored values. The sampled harmonic injections resulting in the minimum absolute error at all frequencies are taken as the final estimated spectra. A final harmonic load flow is performed based on the final estimated spectra to establish the estimated harmonic levels at all nodes. The flow chart of the developed methodology is shown in Figure 9.

\section{Probabilistic assessment with capacitor connected}

To study the effects of the presence of large capacitors in the test feeder on the accuracy of harmonic assessment, a capacitor is connected at the end of the feeder. Different types of capacitor connections, tuned and detuned capacitor banks have different impacts on harmonic performance. The worst case scenario is the grounded $\mathrm{Y}$ connected capacitors connected at the end of the feeder (weakest bus). This type of connection affects even the zero sequence harmonics, and the high network impedance $\mathrm{X}_{\mathrm{S}}$ (weak bus) with large size capacitor connected to it (low $\left.\mathrm{X}_{\mathrm{C}}\right)$ might lead to a resonant condition at low frequencies. A $600 \mathrm{kVAr}$ capacitor, a relatively large capacitor compared to the feeder total reactive load, is connected at Bus 35 (end of the feeder).

The presence of capacitors in the feeder affects the assessment based on the readings at the head of the feeder only. The harmonic currents seen at the head of the feeder are not the total harmonic currents injected by the sources, as the capacitors provide another low impedance paths in addition to the substation. A second monitor is therefore needed at the capacitor bus, to record the harmonic currents flushed through the capacitor. The initial educated guess of currents injected by the load can be done based on the magnitude difference between the substation readings and the capacitor readings. The initial spectra calculated and used as average for the probabilistic assessment is calculated as shown by (3)

$$
\text { Spectra }_{a v r}=\frac{I_{s u b, h}-I_{c a p, h}}{\sqrt{I_{s u b, 1}^{2}+I_{c a p, 1}^{2}}} \times 100 \%
$$

where:

$I_{s u b, h}=$ substation monitor $h_{t h}$ harmonic current reading, $I_{c a p, h}=$ capacitor monitor $h_{t h}$ harmonic current reading, $I_{\text {sub }, 1}=$ substation monitor fundamental current reading, $I_{c a p, l}=$ capacitor monitor fundamental current reading.

Note that the denominator in (3) is an approximate estimate of the total fundamental load current, assuming that the fundamental current at the substation and at the capacitor are perpendicular (i.e., due to highly MVAr compensated feeder the current angle at the substation is approximately $0^{\circ}$ ). 


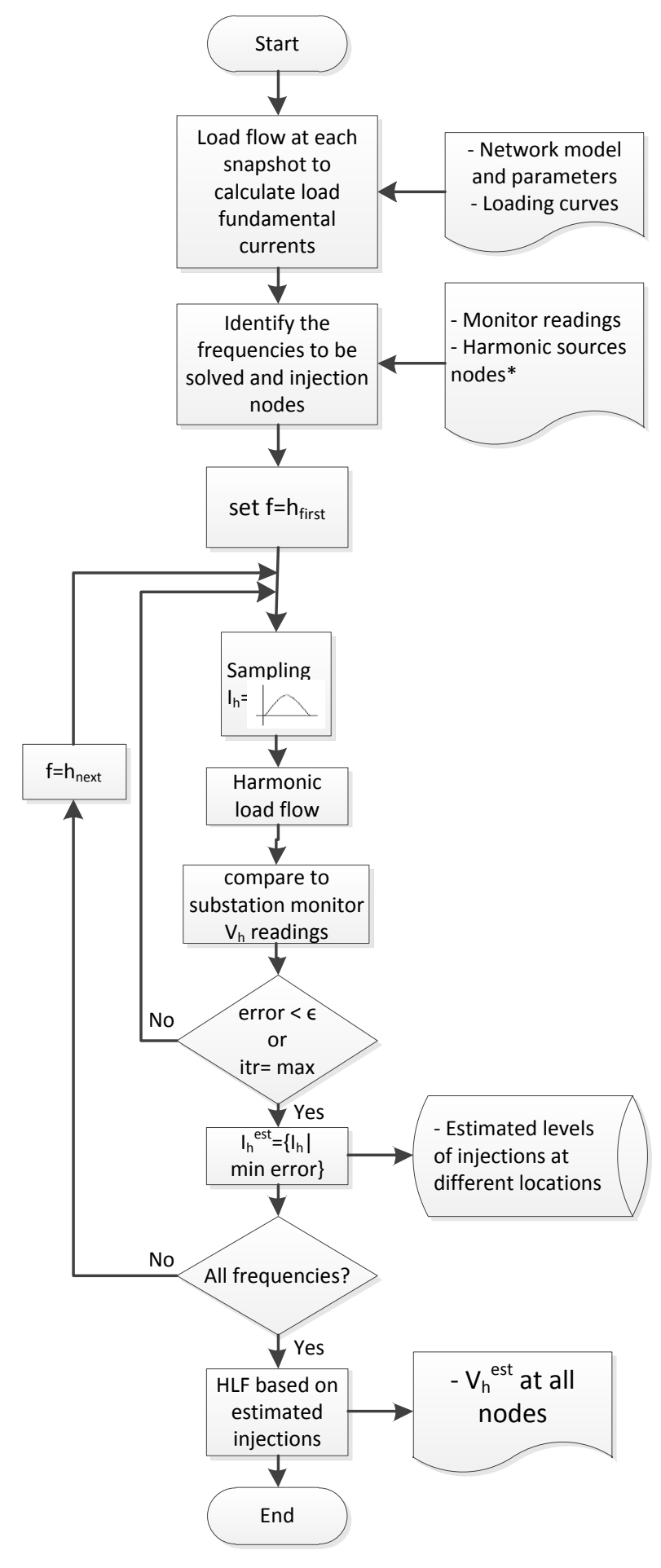

* All nodes with loads are assumed to be locations of harmonic sources

Fig. 9. Flow chart of probabilistic estimation methodology

\section{RESULTS AND ANALYSIS}

All the studies discussed in the previous section are performed per phase. The results shown here are for phase A only for the sake of simplified presentations.

\section{A. Coarse, approximate, analysis}

The results of the impact of increasing the injection at certain points of the feeder on the whole feeder are shown in Figure 10. As can be noted from the figure, the increase of the

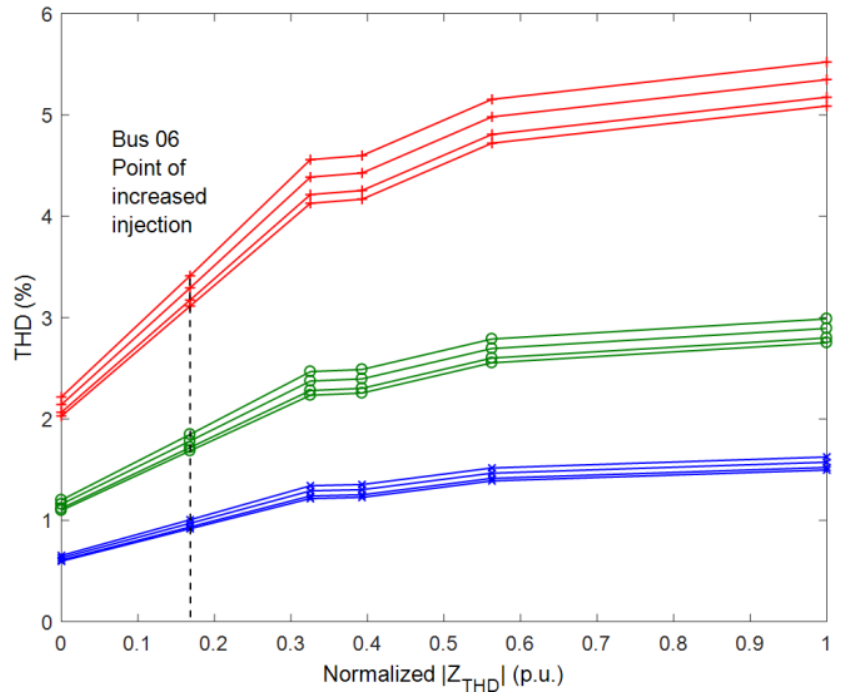

Fig. 10. Impacts on harmonic performance (minimum, average and maximum THD) of the feeder by increased harmonic injection at Bus 06

harmonic injection at one location has effects on the whole feeder with a more pronounced impact at the buses further from the substation. The impact on the point of increased injections (Bus 06) is an increase in the maximum THD from $3.11 \%$ in the generic case study to $3.17 \%$ when the injection is doubled, $3.3 \%$ when the injection is tripled and $3.41 \%$ when the injection is fourfold the original injection, i.e., almost negligible considering the level of increase of harmonic injections. Comparing the impact at the substation and at the furthest bus down the feeder, shows the more pronounced impact at the weaker buses, although they are electrically further from the point of harmonic injection. Bus 01 shows an increase in the maximum THD from $2.03 \%$ to $2.22 \%$ between the original and the fourfold increase in harmonic injection while the furthest bus, Bus 35, shows an increase in the maximum THD from $5.09 \%$ to $5.52 \%$. This shows almost the same ratio of increase, i.e., $10 \%$ of the original values.

The second study was to roughly assess the daily harmonic performance of the feeder based on the readings at the substation and utilising the smoothed generic THD vs. an electrical distance curve. The coefficient of variation calculated at the substation is assumed to be constant along the feeder. Based on the corrected THD values and the constant coefficient of variation, the generic curves were rescaled and the rough estimate was produced. Figure 11 shows the results of the coarse assessment (smoothed blue curves) compared to the true general case THD values for different considered indices. It can be seen that the coarse assessment results in a very accurate estimation of the $95^{\text {th }}$ percentile values. The accuracy of the coarse estimation of maximum THD values is lower though, and in particular for buses further away from the monitoring point. The assessed maximum THD tends to be lower than the actual max THD.

\section{B. Probabilistic assessment}

The results of the one day probabilistic assessment are presented using cumulative distribution functions (CDF) and box plots for the 144 reading samples (6 readings every hour for 24 hours). 


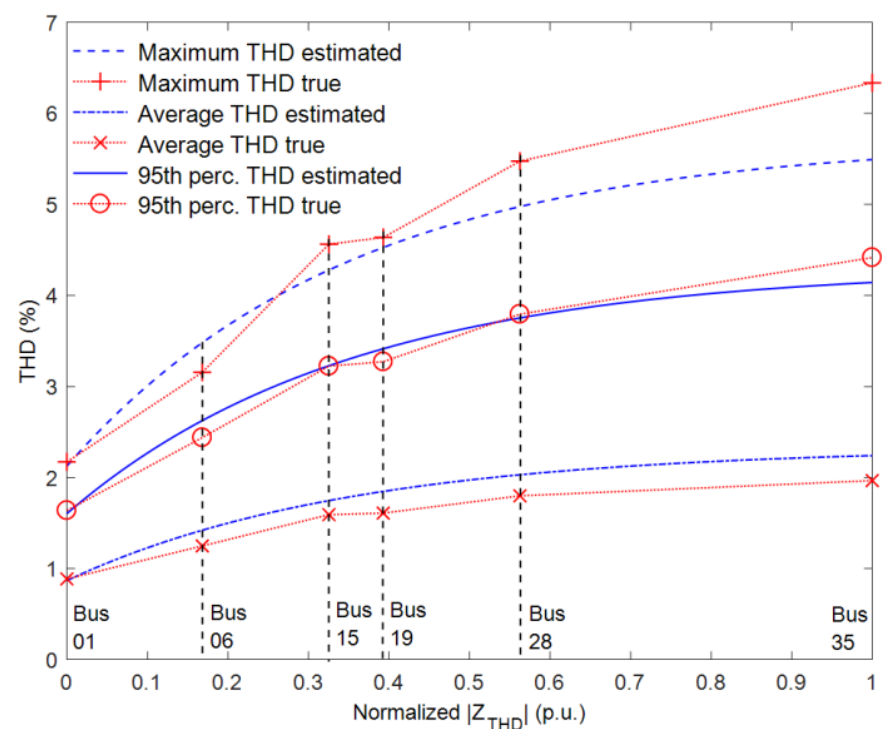

Fig. 11. Coarse assessment for the general case

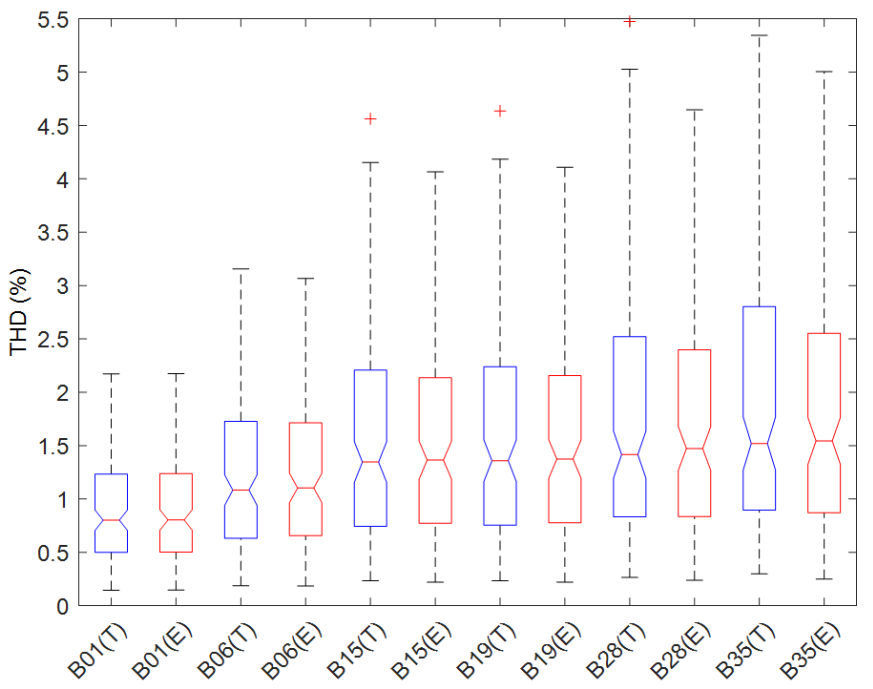

Fig. 12. True(T)/Estimate(E) harmonic performance for the general case study

Figure 12 shows the true (blue) and assessed (red) day performance box plots of the characteristic buses. As shown in the figure, the THD values at Bus 28 and Bus 35 are underestimated considering both the adopted performance indices (average, percentiles and maximum values) and variation ranges (percentile range and total range). This is due to the premature convergence of the algorithm solution (Figure 9), i.e. the error threshold is met at the substation before reaching the best estimate at all buses, especially the more distant buses. The error of the estimate is expressed with respect to the true values.

Figure 13 shows the assessment error samples, i.e., each error reading during the day is recorded and the sample is plotted as a box plot. As shown in the figure, although the average error for the worst performing bus, Bus 35 is less than $12 \%$, individual error readings could reach up to $40 \%$. This is mainly due to harmonic current cancellation, which leads to low current and voltage readings at the substation while higher levels of harmonics are present in the feeder.

The harmonic performance CDFs of three characteristic buses are shown in Figure 14 for both true and assessed daily

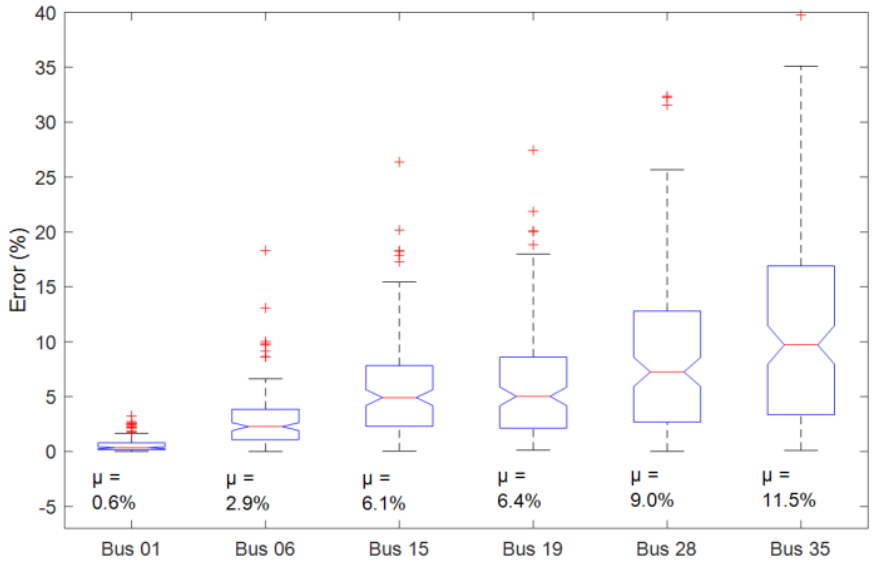

Fig. 13. Estimation errors for the probabilistic assessment

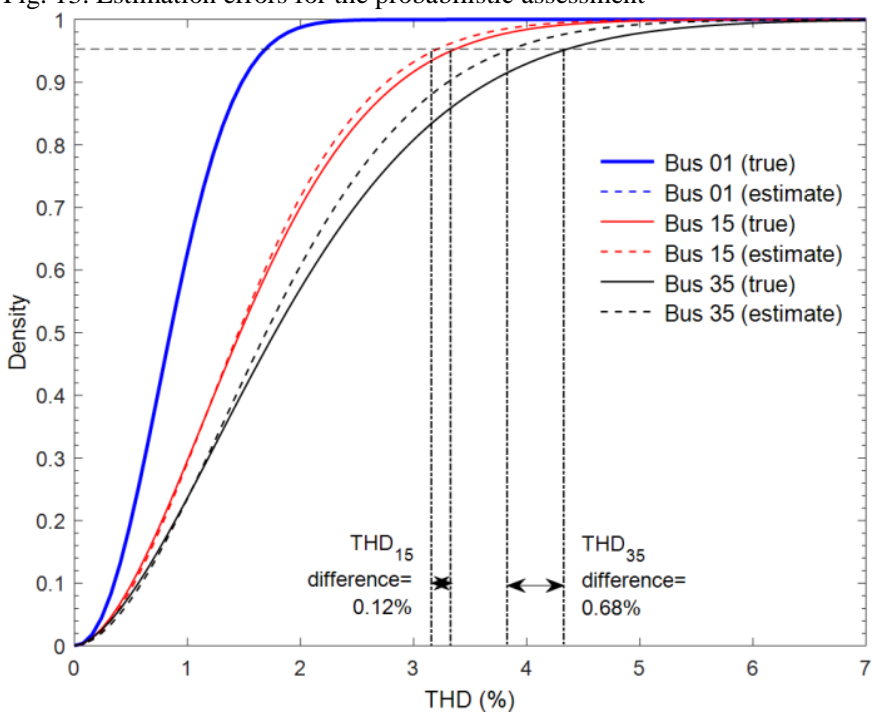

Fig. 14. CDF of harmonic performance for buses 1,15 and 35
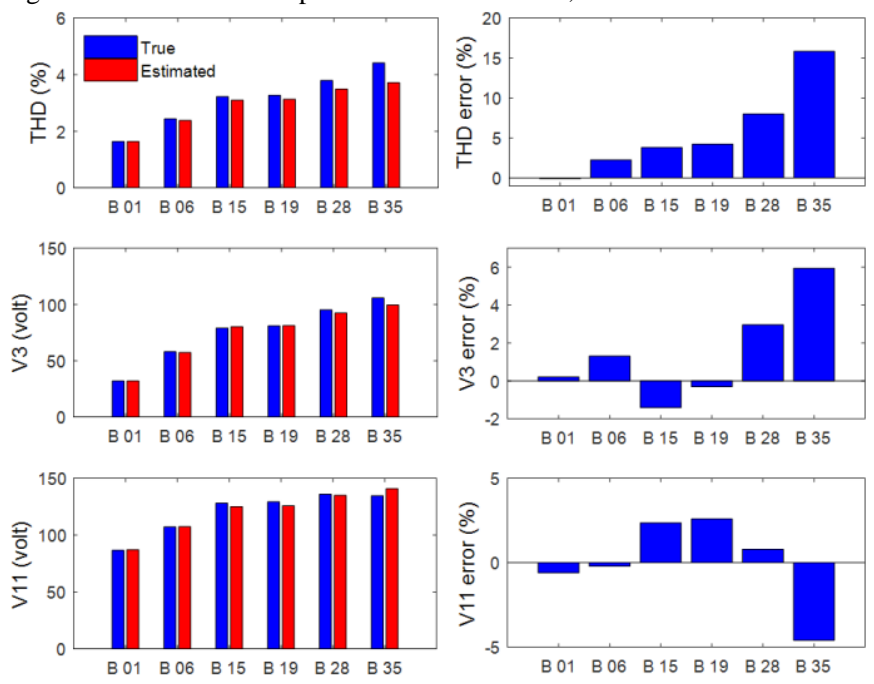

Fig. 15. Comparison of $95^{\text {th }}$ percentile THD values, $3^{\text {rd }}$ and $11^{\text {th }}$ harmonic voltage values and corresponding assessment errors for the characterstic buses

harmonic performance. The discrepancy between true (solid) and estimated (dashed) is the most evident (though still small) for buses 15 and 35 while for Bus 01 the CDFs are identical. Figure 15 gives a closer look into $95^{\text {th }}$ percentiles values for THD and the $3^{\text {rd }}$ and $11^{\text {th }}$ harmonic voltages and corresponding assessment errors. 


\section{Probabilistic assessment with capacitor connected}

For the case of a large capacitor connected at a weak bus, the result of estimation based on the readings at the head of the feeder is shown in Figure 16. As it can be seen the errors of the THD estimation are unacceptable as they exceed $40 \%$ when the capacitor bus information is not included in the evaluation. This is due to the fact that: i) a high portion of the harmonic currents does not reach the substation; ii) the resonance at the $9^{\text {th }}$ harmonic is excited due to the size of the capacitor, location and connection type. With a second monitor connected at the capacitor bus, the initial values of the loads spectra for the probabilistic evaluation are calculated based on equation (3). By including the extra monitor information, the assessment for all harmonic voltages except at the resonant frequency is significantly improved. As shown in Fig. 17, the errors in estimating the $95^{\text {th }}$ percentiles of the $11^{\text {th }}$ and the $13^{\text {th }}$ harmonic voltages are substantially reduced and they are now less than 5\%. However, due to the resonance at the $9^{\text {th }}$ harmonic, the assessment at this frequency is still inaccurate regardless of the extra information provided by additional monitor. The errors in harmonic assessment are in this case around $30 \%$, and this high error is subsequently transferred to the calculated THD, resulting in errors in the range of $20-30 \%$.

\section{Discussion}

The main limitation of the proposed probabilistic assessment methodology based on substation readings occurs in cases where harmonic currents do not reach the substation. This can happen mainly when either the lower impedance path is available through filters and power factor correction capacitors, or out of phase currents are injected at different nodes, which leads to a high level of cancellation. The former can be addressed by additional harmonic monitoring at the capacitor node as shown in Section V.C. The latter is expected to happen less in radial feeders as suggested in [16].
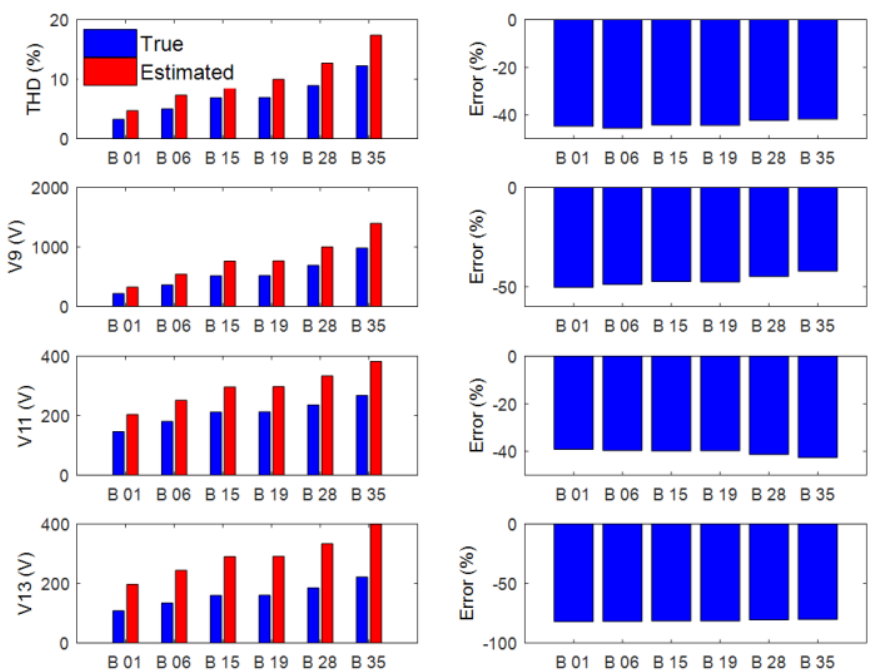

Fig. 16. The $95^{\text {th }}$ percentiles of THD, $9^{\text {th }}, 11^{\text {th }}$ and $13^{\text {th }}$ harmonic voltages and corresponding estimation errors - non monitored capacitor at Bus 35
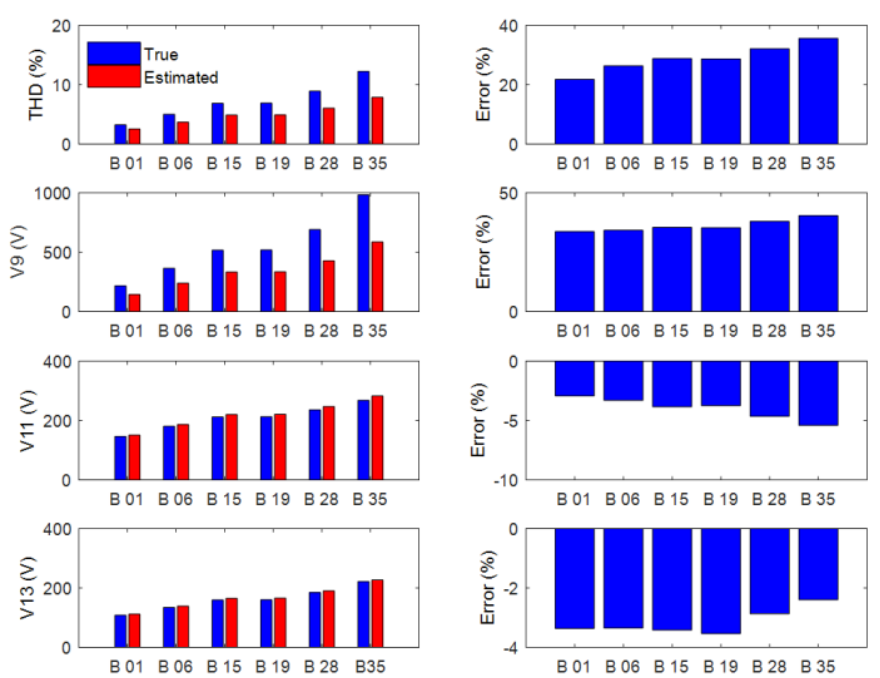

Fig. 17. The $95^{\text {th }}$ percentile THD, $9^{\text {th }}, 11^{\text {th }}$ and $13^{\text {th }}$ harmonic voltages and corresponding estimation errors - monitored capacitor at Bus 35

It is also suggested in [1] to use a practical summation exponent $(\alpha)$, ranging from 1 to 2 in equation (4). for the aggregation of $i$ harmonic voltages $\left(V_{h i}\right)$ in order to calculate the resultant voltage $\left(V_{h}\right)$ in the second summation law in [1]. The expected phase angle between harmonic sources varies between $0^{\circ}$ (linear summation) and $90^{\circ}$ (quadratic summation).

$$
V_{h}=\alpha \sqrt{\sum_{i}\left(V_{h i}^{\alpha}\right)}
$$

This methodology also fails to assess harmonic voltages at the resonance frequencies. However, the harmonic resonance is a special case which the DSO should prevent by adopting appropriate measures at network design stage and while commissioning capacitors and transformers. If it occurs however, the protection systems of the shunt devices should operate (due to recorded high currents or voltages) and isolate the device and hence remove the cause of the resonance.

\section{CONCLUSION}

Considering that the accurate knowledge of harmonic levels at distribution level buses is still not required by electricity market regulators in many countries, except for facilitating new load and generation connections, this paper presented simple, effective and sufficiently accurate methodologies for the assessment of harmonic levels in radial feeders based on harmonic monitoring at a substation. The approximate (coarse) assessment of harmonic levels can be performed using look-up curves developed and based on the electrical distance to the monitored bus. The more elaborate, probabilistic assessment can provide the THD and harmonic voltage levels at all buses along the feeder with errors in $95^{\text {th }}$ percentile values of THD below $15 \%$ and of individual harmonic voltages below $10 \%$.

Having in mind the trend towards integrating more renewables at the distribution level proliferation of power electronics, interfaced load and generation and storage technologies, and the increased attention on power quality performance, the ability to quickly assess the harmonic performance of the network, based on existing limited 
monitoring, can prove essential for the planning of cleaner and more flexible distribution networks. In this context, the proposed methodology can be used very effectively as it provides a simple and cost effective way to indicate the level of DG penetration, load growth and new load connections that might lead to violation of harmonic planning levels. It can also identify, based on simple estimates and look up curves, locations along the feeder where further more accurate harmonic analysis should be performed.

The main limitation of the presented probabilistic approach is the overestimation of harmonic performance when large capacitors are connected at the end of the feeder. Additional monitors at the capacitor nodes could solve this issue, however, the harmonic voltages at resonant frequencies would still not be assessed accurately. The overall improvement in the accuracy of the assessment could be achieved by considering capacitor size and location as the additional random variables in the Monte Carlo simulation, if the information from additional monitors cannot be readily obtained.

\section{ACKNOWLEDGMENT}

The authors wish to thank the discussion platform of the OpenDSS software, in particular Mr. R. Dugan and Mr. D. Montenegro, for their help and tips with the simulation tool.

\section{REFERENCES}

[1] IEC, "IEC 61000-3-6:1996 - Assessment of emission limits for distorting loads in MV and HV power systems," 1996.

[2] IEEE, "IEEE 519:2014. IEEE Recommended practices and requirements for harmonic control in electrical power systems," 2014.

[3] ENA, "ER G5/4-1: Planning levels for harmonic voltage distortion and the connection of non-linear equipment to transmission systems and distribution networks in the United Kingdom”, 2005.

[4] J. E. Farach, W. M. Grady, and A. Arapostathis, "An optimal procedure for placing sensors and estimating the locations of harmonic sources in power systems," IEEE Trans. on Power Del., vol. 8, no. 3, pp. 1303-1310, 1993.

[5] G. T. Heydt, "Identification of harmonic sources by a state estimation technique," IEEE Trans. on Power Del., vol. 4, no. 1, pp. 569-576, 1989.

[6] A. P. S. Meliopoulos, Zhang Fan, and S. Zelingher, "Power system harmonic state estimation," IEEE Trans. on Power Del., vol. 9, no. 3, pp. 1701-1709, 1994.

[7] C. Rakpenthai, S. Uatrongjit, N. R. Watson, and S. Premrudeepreechacharn, "On harmonic state estimation of power system with uncertain network parameters," IEEE Trans. on Power Syst., vol. 28, no. 4, pp. 4829-4838, 2013.

[8] N. R. Watson, "Power Quality State Estimation", European Trans. Electrical Power; vol. 20, No 1, Jan. 2010, pp. 19-33.

[9] I. Molina-Moreno, A. Medina, R. Cisneros-Magaña, O. AnayaLara, "Time Domain Harmonic State Estimation in Unbalanced Power Networks based on Optimal Number of Meters and the Principle of Half-Wave Symmetry", IET Proceedings on Generation, Transmission and Distribution, Vol. 11, Issue 15, Oct. 2017, pp. 3871-3880.

[10] R. Cisneros-Magaña, A. Medina, V. Dinavahi, A. Ramos-Paz, "Time-domain power quality state estimation based on Kalman filter using parallel computing on graphics processing units", IEEE Access, Vol. 6, Issue 1, April 2018, pp. 21152-21163.
[11] A. Arefi, M. R. Haghifam, and S. H. Fathi, "Distribution harmonic state estimation based on a modified PSO considering parameters uncertainty," in Proc. IEEE PowerTech conf.,Trondheim, 2011, pp. 1-7.

[12] G. D'Antona, C. Muscas, and S. Sulis, "State estimation for the localization of harmonic sources in electric distribution systems," in Proc. IEEE Instrumentation and Measurement Technology Conf., IMTC, , 2008, pp. 865-869.

[13] N. Okada and K. Yukihira, "Harmonic state estimation in distribution network based on fifth harmonic current characteristic," in Proc. IEEE 16th Int. Conf. on Harmonics and Quality of Power (ICHQP), 2014, pp. 566-570.

[14] Mark F. McGranaghan Roger C. Dugan, Surya Santoso, H. Wayne Beaty, Electrical Power Systems Quality, 2nd ed. New York: McGraw-Hill, 2002

[15] ELECTROTEK CONCEPTS, "Reliability benchmarking methodology," EPRI 1997.

[16] T. Hiyama, M. S. A. A. Hammam, and T. H. Ortmeyer, "Distribution system modeling with distributed harmonic sources," IEEE Trans. on Power Del., vol. 4, no. 2, pp. 1297 1304, 1989.

[17] M. F. McGranaghan, R. C. Dugan, Jack A. King, and W. T. Jewell, "Distribution Feeder Harmonic Study Methodology," IEEE Trans. on Power Apparatus and Syst., vol. PAS-103, no. 12, pp. 3663-3671, 1984.

[18] A. E. Emanuel, J. Janczak, D. J. Pileggi, E. M. Gulachenski, C. E. Root, M. Breen, and T. J. Gentile, "Voltage distortion in distribution feeders with nonlinear loads," IEEE Trans. on Power Del., vol. 9, no. 1, pp. 79-87, 1994.

[19] I. N. Santos, V. Cuk, P. M. Almeida, M. H. J. Bollen, and P. F. Ribeiro, "Considerations on hosting capacity for harmonic distortions on transmission and distribution systems," EPSR, no. 119, pp. 199-206, 2015.

[20] A. Bhowmik, A. Maitra, S. M. Halpin, and J. E. Schatz, "Determination of allowable penetration levels of distributed generation resources based on harmonic limit considerations," IEEE Trans. on Power Del., vol. 18, no. 2, pp. 619-624, 2003.

[21] T. H. Ortmeyer and T. Hiyama, "Distribution system harmonic filter planning," IEEE Trans. on Power Del., vol. 11, no. 4, pp. 2005-2012, 1996.

[22] SuSTAINABLE Deliverable 2.2, "SuSTAINABLE validation scenarios definition", 2014.

[23] SuSTAINABLE Deliverable 3.6, "Methodology for provision of differentiated QoS", 2015.

[24] George J. Wakileh, Power Systems Harmonics: Fundamentals, Analysis and Filter Design. New York: Springer, 2001

Sami Abdelrahman received his MSc and $\mathrm{PhD}$ degrees from the University of Manchester, Manchester, UK in 2012 and 2016 respectively. From 2009 to 2011, he worked with National Electricity Corporation, Sudan Currently he is with Network Development Department in the Nationalgrid ESO, Warwick, UK.

Jovica V. Milanović (M'95, SM'98, F'10) received his PhD degree from the University of Newcastle, Australia, and his Higher Doctorate (DSc degree) from The University of Manchester, UK, all in Electrical Engineering. Currently, he is a Professor of electrical power engineering, Deputy Head of School and Director of External Affairs with the School of Electrical and Electronic Engineering at The University of Manchester, UK. Professor Milanovic is a Chartered Engineer in the UK, Foreign member of the Serbian Academy of Engineering Sciences, Fellow of the IET, Fellow of the IEEE, Distinguished IEEE PES Lecturer and currently serves on IEEE PES Governing Board as Regional Representative for Europe, Middle east and Africa and as a vice-chair of the IEEE PES Fellows Committee. 